\title{
Consumers' Purchase Intentions of Shoes: Theory of Planned Behavior and Desired Attributes
}

\author{
Yun Wang ${ }^{1}$ \\ ${ }^{1}$ Department of Fashion Design and Management, National Pingtung University of Science and Technology, \\ Taiwan, R.O.C. \\ Correspondence: Yun Wang, No. 1 Shuehfu Rd., Neipu, Pingtung, Taiwan, R.O.C. Tel: 886-8-770-3202 ext. \\ 7543.E-mail:yunw@mail.npust.edu.tw
}

$\begin{array}{lcc}\text { Received: April 3, } 2014 & \text { Accepted: April 23, } 2014 & \text { Online Published: July 28, } 2014 \\ \text { doi:10.5539/ijms.v6n4p50 } & \text { URL: http://dx.doi.org/10.5539/ijms.v6n4p50 }\end{array}$

\begin{abstract}
Nowadays, females viewed shoes are not considered just footwear to protect and comfort foot, but a fashion product for decoration and express self-image. The total annual sale of shoes is NT\$ 60 billion in which female shoes accounted for about NT\$36 billionin in Taiwan. The purpose of this research was to investigate what factors make the difference in female shoes purchase intentions? Factors included (1) shoes attributes; (2) attitude, subjective norm, and perceived behavioral control based on Theory of Planned Behavior; and (3) demographic and shopping behavior variables. A total of 450 convenience questionnaires were distributed outside department stores using Mall-intercept method in Kaohsiung, Taiwan. The results indicated female consumers who have higher purchase intentions of shoes have significant higher appraisal of shoes attributes in style, colour, collocability, materials and brand name compare to those who have lower purchase intentions of shoes. In addition, consumers who have higher purchase intentions of shoes have better attitude, subjective norm, and behavior control compare to those who have lower purchase intentions of shoes. Overall, youth female consumers age between 18-35 years old have significant higher purchase intentions than the elder consumers. Additionally, the more shoes quantity and higher shoes purchase frequency consumers have, the higher purchase intentions of shoes would perceived.
\end{abstract}

Keywords: shoes attributes, Theory of Planned Behavior, purchase intention, attitude, subjective norms, perceived behavior control

\section{Introduction and Research Framework}

Nowadays, females viewed shoes are not considered just footwear to protect and comfort foot, but a fashion product for decoration and express self-image. Gobi International (2010) market research indicated the global footwear market is reach US\$ 174.8 billion in year 2010, and expects to reach US\$ 195.6 billion and have $2.4 \%$ compound annual growth rate (CAGR) in year 2011 to 2015. In Taiwan, footwear market sales grasp NT\$ 67 billion in year 2011; and female shoes sales account for almost NT $\$ 36$ billion (54\%) compare to male (36\%) and children (10\%) (Industrial Development Bureau, R. O. C., 2011). According to Executive Yuan, R. O. C. (2013), Taiwan Female labor force participation rate is excess of 50\% in the year of 2012. Female labor amount was 4.61 million in 2010 and increased to 4.77 million in 2012. Hedrick-Wong (2007) asserts in year 2014 female consumers in affluent countries of Asia hold the consumption power of US\$ 516 billion, and women in developing countries have US\$ 181 billion. Even though government data and online market research analysts indicated shoes consumption is important in daily life of women, there is very limited research that was conducted specifically on shoes worldwide.

The Lancaster's theory of consumer choice (1966) postulates that consumption decisions are determined by the utility that is derived from the attributes of a good. Because consumers often link attributes to consequences of purchasing or consuming products (Akpoyomare, Adeosun, \& Ganiyu, 2012), marketers differentiate and set its product apart from competitors based on a specific attributes (Belch \& Belch, 2001). A research by Forney, Park and Brandon (2005) found that image, quality, colour/style, and design/beauty of fashion products are important criteriawhen purchasing extended brands of casual apparel and home furnishings. Saha, Dey, and Bhattacharaya (2010) applied the 4Ps of marketing mix as a framework to classify and evaluate the importance level of shoes attributes in Kolkata, India. They found that the factors to be considered by shoes manufacturers and marketers 
are "quality, durability, right pricing, after sale service, and convenient location of the retail shops". Laiwechpittaya and Udomkit (2013) expanded the research of Saha, Dey, Bhattacharyya (2010) byadding retail mix (6Ps) with additional factors on presentation and personnel. In their research they found among 50 shoes attributes, most desired attributed for consumers are "comfort, durability, odorless, value, and quality" in Thailand. In second stage, these 50 attributes were grouped into 5 factors using Exploratory Factor Analysis and named as "well trained and experienced salesforce", "product quality and functions", "attractive store and product presentation", "price and perceived value", "health and comfort", and "fashion and trends". According to Aaker, Batra, and Myers, (1992) an important attribute is one that offers an important benefit towards the satisfaction of consumer needs. Aaker (1991) adds that because most product attributes provide consumer benefits, there is usually a one-to-one comparative between brands. Kotler (2002) explains that the most salient attributes or features of a product to the consumer do not necessarily always imply that they are the most important ones. Manufacturers must view product attributes from consumers'point, understand their specific needs to reduce the lost opportunity to sell or raise the level of customer satisfaction. Therefore, identification of those important factors will help the shoe-making companies to tailor their marketing and manufacturing strategies to take advantage of these influences in a way that will satisfy both the consumers and marketers.

The Theory of Planned Behavior (TPB) one of the most widely researched models forpredicting behavioral intentions by social psychologists (Armitage \& Conner, 2001), suggests that beliefs and their corresponding attitudes, subjective norms, and perceivedbehavioral control influence intentions, which drive actual behavior. Attitude (ATT) is defined as the psychological emotion and the positive or negative evaluation that arise when an individual engagesin certain behaviors (Eagly \& Chaiken, 1993). Subjective norms (SN) are the perceived opinions of significant others who are close/important to an individual and who influencehis/her decision-making (Ajzen, 1991). Perceived behavioral control (BC) refers to an individual's perception of the possible difficulties when performing aspecific behavior (Ajzen, 1991). Researchers have most frequently relied on the TPB to explain various consumer issues on ethical (Culiberg, 2014; Shaw \& Shiu, 2002), smoking (Topa\&Moriano, 2010), speed driving (Elliott, Armitage, \&Baughan, 2007), and drug abuse (Bashirian, Hidarnia, Allahverdipour, \& Hajizadeh, 2012). In fashion field, Kim and Karpova (2010) examined US female college students' intent to purchase fashion counterfeits based on TPB. They found product appearance, past purchase behavior, value consciousness, and normative susceptibility are significant predictors of attitude toward buying fashion counterfeit goods. Attitude, subjective norms, and perceived behavioral control are significantly related to intent to purchase fashion counterfeit goods. Ling (2009) examine luxury fashion goods purchase intention in China by applying the TPB with an additional factor, cultural orientation. He found the three factors of TPB, attitude, subjective norms, and perceived behavioral control have significant effects on luxury fashion goods purchase intention, but not cultural orientation.

The purpose of this research was to investigate what factors make the difference in female shoes purchase intentions? Factors included (1) shoes attributes: style, colour, material, brand, collocability, comfort, durability, and service; (2) attitude, subjective norm, and perceived behavioral control based on TPB; and (3) demographic and shopping behavior variables: age, income, education level, occupation, marital status, quantity of shoes own, shoes purchased frequency, average purchased price of shoes, purchased locations, and shopping frequency. The following hypotheses were proposed:

H1.Consumers who have higher purchase intentions of shoes will differ significantly in shoes attributes appraisal from those consumers who have lower purchase intentions of shoes.

H2.Consumers who have higher purchase intentions of shoes will differ significantly in attitude, subjective norms, and perceived behavioral control from those consumers who have lower purchase intentions of shoes.

H3a.Consumers'demographic variables have significant effects on their preference of shoes attributes and purchase intention.

H3b.Consumers' shopping behavior variables have significant effects on their preference of shoes attributes and purchase intention.

\section{Methodology}

\subsection{Sample and Data Collection}

Prior studies have shown the importance of gender effect (Davis, Lang, \& San Diego, 2014) in retail shopping behavior. Therefore, the sampling for this research is mainly female consumers. Data were collect by using survey questionnaire, in the beginning of questionnaire; information of fashion shoes that are not counting sneakers and slippers was given. There are three parts of questionnaires: (1) demographic and shopping behavior 
variables; (2) shoes attributes; and (3) attitude, subjective norms, consumer perceived behavior control and purchase intention (PI) of fashion shoes. Consumers' belief of fashion shoes attributes was operationalized based on Stanton, Michael and Bruce (1991) definition of product attributes, Chang, Wang, and Cheng (2010) research of sport shoes attributes, Laiwechpittaya and Udomkit (2013), and interviewed five fashion shoes sales and ten female consumers in Taiwan. An 8-items scale of fashion shoes attributes was produced at the end. Attitude, subjective norms, and consumer perceived behavior control were measured on the basis of Canniere, Pelsmacker and Geuens (2009). Purchase intention (PI) was measured using three items adapted from Bhattacherjee (2000). All measurements used a 7-point Likert scale with response options ranging from 1-strongly disagree to 7-strongly agree. A pilot study including sixty-six female customers was conducted to test the clarity of questionnaire wording. Using Mall-intercept method we distributed 450 questionnaires to customers of four department stores during the months of October and November, 2013. These department stores locate in Kaohsiung, a Southern city in Taiwan. The final data analysis included 422 completedquestionnaires and the overall response rate was $94 \%$.

\subsection{Data Analysis}

Data were analyzed by SPSS 17.0. In order to test research hypotheses, respondents were classified into two groups based on the difference between their mean scores on purchase intentions of fashion shoes. A cluster analysis was performed to help classify respondents into more homogeneous preference groups. This approach has been conducted by various researchers in order to determine customer segments based on distinct preference profiles (Head \& Ziolkowski, 2012). The $k$-means cluster procedure in SPSS was used to perform the segmentation. An analysis of variance revealed that the segments in the 2-cluster solution differed significantly $(\mathrm{F}=4.73, \mathrm{p}<.001)$ from low and high purchase intentions of fashion shoes. Cluster 1 is the low purchase intentions of fashion shoes group $(\mathrm{n}=142, \mathrm{Mean}=4.20, \mathrm{SD}=.75)$, and cluster 2 is the high purchase intention of fashion shoes group $(n=208$, Mean=6.24, $\mathrm{SD}=.55)$. After these low and high purchase intentions groups were identified, the independent $t$-test analysis ofvariance for groups mean difference was performed to test hypotheses 1 and 2. Afterward, one factor analysis of variance (ANOVA) was apply to examine hypotheses 3a and $3 b$, differences in consumers'demographic and shopping behavior variables in their preference of shoes attributes and purchase intentions.

\section{Results and Analysis}

\subsection{Reliability and Descriptive Analysis of Respondents}

Internal reliability was identified by calculating the Cronbach's alphas, which ranged from 0.77 (shoes attributes), 0.80(BC), $0.84(\mathrm{SN}), 0.89$ (ATT), to 0.91(PI), indicates our research variables have good internal consistency reliability. The majority of respondents are females age between $26-35$ years old (40.5\%), haveeducation of college degree (54.7\%) and with monthly income around NT $\$ 20,001 \sim 30,000(39.3 \%)$. Detail information of the study population was given in Table 1.

\subsection{Differences between Low and High Purchase Intentions in Shoes Attributes}

The results of independent $t$-test showed the differences between low and high fashion shoes purchase intentions in each attribute of shoes were displayed in Table 2. Two important facts were derived. First, among these shoes attributes, female consumers rank the highest importance in comfort (Mean=6.48), follow by style (Mean=6.33), colour (Mean=6.19), shoes materials (Mean=6.15) and collocability (Mean=6.14) attributes of shoes in Taiwan. Compare the results with Laiwechpittaya and Udomkit (2013) research in Thailand unisex shoes market, both research initiated "comfort" is the most important attribute for consumers while evaluate the shoes product. However, Thailand consumers evaluated the durability, odorless, value for price paid and high quality as the following important desired attributes of shoes which are not the same as Taiwan female consumers' perspective. Second, results in Table 2 suggested our hypothesis 1, consumers who have higher purchase intentions of shoes differ significantly in shoes attributes appraisal from those consumers who have lower purchase intentions of shoes, is partial supported. Female consumers who have higher purchase intentions of shoes have significant higher appraisal of shoes attributes in style, colour, collocability (in $\mathrm{p}<.001$ level), materials and brand name (in $\mathrm{p}<.01$ level) compare to those who have lower purchase intentions of shoes. However, no significant differences were found between those high and low groups in comfort, durability and service attributes of shoes. Meaning even though "comfort, durability, and service" are important attributes in shoes market from most consumers' and shoes manufactures perspective, these factors are the minimum essential attributes of shoes; the "style, colour, collocability, material, and brand name" are the real motivated attributes trigger female consumers' purchase intentions of shoes. 
Table 1. Profile of the study population

\begin{tabular}{|c|c|c|c|}
\hline Variable & Category & Frequency & $\%$ \\
\hline \multirow[t]{4}{*}{ Age } & $18 \sim 25$ & 106 & 25.1 \\
\hline & $26 \sim 35$ & 171 & 40.5 \\
\hline & $36 \sim 45$ & 117 & 27.7 \\
\hline & Over 45 & 28 & 6.6 \\
\hline \multirow[t]{4}{*}{ Education } & High school & 76 & 18.0 \\
\hline & Junior College & 56 & 13.3 \\
\hline & College/University & 231 & 54.7 \\
\hline & Graduate & 59 & 14.0 \\
\hline \multirow[t]{7}{*}{ Occupation } & Students & 35 & 8.3 \\
\hline & Public servant ${ }^{\mathrm{a}}$ & 28 & 6.6 \\
\hline & Manufacture industry & 68 & 16.1 \\
\hline & Service industry & 186 & 44.1 \\
\hline & Soho & 35 & 8.3 \\
\hline & Household & 11 & 2.6 \\
\hline & Others & 59 & 14.0 \\
\hline Monthly & $<\mathrm{NT} \$ 20,000^{\mathrm{b}}$ & 79 & 18.7 \\
\hline \multirow[t]{4}{*}{ income } & NT\$ $20,001-30,000$ & 166 & 39.3 \\
\hline & NT\$ $30,001-40,000$ & 96 & 22.7 \\
\hline & NT\$ 40,001-50,000 & 48 & 11.4 \\
\hline & $>$ NT\$ 50,001 & 33 & 7.8 \\
\hline Marital & Single & 244 & 57.8 \\
\hline \multirow[t]{2}{*}{ status } & Married & 171 & 40.5 \\
\hline & Divorced & 7 & 1.7 \\
\hline \multirow[t]{5}{*}{ Shoes QTY } & 1 pairs & 18 & 4.3 \\
\hline & $2 \sim 4$ pairs & 134 & 31.8 \\
\hline & $5 \sim 7$ pairs & 108 & 25.6 \\
\hline & $8 \sim 10$ pairs & 73 & 17.3 \\
\hline & $>11$ pairs & 89 & 21.1 \\
\hline Shoes & A month & 33 & 7.8 \\
\hline purchased & 3 months & 154 & 36.5 \\
\hline \multirow[t]{3}{*}{ frequency } & 6 months & 151 & 35.8 \\
\hline & A year & 58 & 13.7 \\
\hline & Over a year & 26 & 6.2 \\
\hline Average & $<\mathrm{NT} \$ 1,500$ & 152 & 36.0 \\
\hline purchased price & NT\$1,501 2,500 & 187 & 44.3 \\
\hline \multirow[t]{3}{*}{ of shoes } & $\mathrm{NT} \$ 2,501 \sim 3,500$ & 57 & 13.5 \\
\hline & NT\$3,501 4,500 & 14 & 3.3 \\
\hline & $>\mathrm{NT} \$ 4,500$ & 12 & 2.8 \\
\hline Last time & Dept. stores & 199 & 47.2 \\
\hline purchased & Retail stores & 149 & 35.3 \\
\hline \multirow[t]{3}{*}{ Location } & Street vendors & 12 & 2.8 \\
\hline & Online stores & 54 & 12.8 \\
\hline & Night markets & 8 & 1.9 \\
\hline
\end{tabular}

Note. $\mathrm{n}=422$; ${ }^{\mathrm{a}}$ Including army/government official /public school teachers; ${ }^{\mathrm{b}} \mathrm{NT} \$$, new Taiwan dollars; US\$1 $=\mathrm{NT} \$ 29.35$ in October, 2013.

Table 2. Independent $t$-test between low and high purchase intentions in shoes attributes

\begin{tabular}{|c|c|c|c|c|c|c|c|c|c|}
\hline \multirow[t]{2}{*}{ Shoes attributes } & \multirow[t]{2}{*}{ Mean } & \multirow[t]{2}{*}{ SD } & \multicolumn{2}{|l|}{ Low PI } & \multicolumn{2}{|c|}{ High PI } & \multirow{2}{*}{$\begin{array}{l}\text { Mean } \\
\text { dif. }\end{array}$} & \multirow[t]{2}{*}{ t-value } & \multirow[t]{2}{*}{$\mathrm{p}$ value } \\
\hline & & & Mean & SD & Mean & SD & & & \\
\hline Style & 6.33 & 0.94 & 5.87 & 1.21 & 6.56 & 0.65 & 0.69 & -7.59 & $.000^{* * *}$ \\
\hline Colour & 6.19 & 0.89 & 5.91 & 1.04 & 6.33 & 0.76 & 0.42 & -4.72 & $.000^{* * *}$ \\
\hline Materials & 6.15 & 0.97 & 5.95 & 1.07 & 6.25 & 0.90 & 0.30 & -2.98 & $.003^{* *}$ \\
\hline Collocability & 6.14 & 0.97 & 5.70 & 1.10 & 6.36 & 0.82 & 0.67 & -7.03 & $.000^{* * *}$ \\
\hline Brand & 4.49 & 1.34 & 4.21 & 1.34 & 4.63 & 1.32 & 0.42 & -3.05 & $.002^{* *}$ \\
\hline Comfort & 6.48 & 0.77 & 6.42 & 0.89 & 6.51 & 0.70 & 0.09 & -1.15 & .250 \\
\hline Durability & 6.01 & 1.03 & 6.01 & 1.05 & 6.02 & 1.03 & 0.01 & -0.1 & .919 \\
\hline Service & 5.47 & 1.25 & 5.42 & 1.37 & 5.50 & 1.19 & 0.08 & -0.6 & .548 \\
\hline
\end{tabular}

Note. $* * p<.01, * * * p<.001$. 


\subsection{Differences between Low and High Purchase Intentions in ATT, SN, and BC}

To examine the hypothesis 2 , independent $t$-test was performed and results were display in Table 3 . Overall most consumers have favorite attitudes toward fashion shoes (Mean= 5.13) and consider themselves have high perceived behavior control of fashion shoes purchase behavior (Mean= 5.41). Results in Table 3 also suggested our hypothesis 2, consumers who have higher purchase intentions of shoes have significant differences in attitude $(t=-11.56, \mathrm{p}<.001)$, subjective norms $(t=-5.82, \mathrm{p}<.001)$, and perceived behavioral control $(t=-8.37$, $\mathrm{p}<.001$ ) from those consumers who have lower purchase intentions of shoes, is supported. Particularly, consumers who have higher purchase intentions of shoes have better attitude (Mean=5.50), higher subjective norms (Mean=4.81) and perceived better behavior control (Mean=5.68) compare to those who have lower purchase intentions of shoes in attitude (Mean=4.39), subjective norm (Mean=4.18), and behavior control (Mean=4.86).

Table 3. Independent $t$-test between low and high purchase intentions in ATT, SN, and BC

\begin{tabular}{|c|c|c|c|c|c|c|c|c|c|}
\hline \multirow[t]{2}{*}{ Variables } & \multirow[t]{2}{*}{ Mean } & \multirow[t]{2}{*}{ SD } & \multicolumn{2}{|c|}{ Low PI } & \multicolumn{2}{|c|}{ High PI } & \multirow[t]{2}{*}{ Mean dif. } & \multirow[t]{2}{*}{$t$ value } & \multirow[t]{2}{*}{$p$ value } \\
\hline & & & Mean & SD & Mean & SD & & & \\
\hline ATT & 5.13 & 1.06 & 4.39 & 1.02 & 5.50 & .87 & -1.10 & -11.56 & $.000^{* * *}$ \\
\hline $\mathrm{SN}$ & 4.60 & 1.10 & 4.18 & 1.11 & 4.81 & 1.03 & -.63 & -5.82 & $.000^{* * *}$ \\
\hline $\mathrm{BC}$ & 5.41 & 1.03 & 4.86 & 1.07 & 5.68 & .89 & -.82 & -8.37 & $.000^{* * *}$ \\
\hline
\end{tabular}

Note. $* * * p .001$.

\subsection{Demographic and Shopping Behavior Variables Differences in Shoes Attribute and Purchase Intentions}

Analysis of variance (ANOVA) was used to examine hypotheses $3 \mathrm{a}$ and $3 \mathrm{~b}$ : consumers' demographic and shopping behavior variables have significant effects on their preference of shoes attributes and purchase intentions. If significant exist, Scheffé post hoc analysis is applied to reveal the difference among variable categories. Results in Table 4 suggested Hypothesis $3 \mathrm{a}$ is partial supported, since the different age, marriage status, and income level of female consumers had significant effects on style, colour, brand name, comfort attributes and purchase intentions of fashion shoes. In general, youth female consumers age between 18-35 years old have significant higher purchase intentions $(\mathrm{F}(3,418)=12.906, \mathrm{p}<.001)$ than elder consumers. To be specific, female consumers age between $26-35$ years old tend to care shoes' style $(F(3,418)=4.320, p<.01)$, colour $(F(3$, $418)=5.780, \mathrm{p}<.01)$ and brand name $(\mathrm{F}(3,418)=3.843, \mathrm{p}<.05)$ more than those female consumers age between $36-45$ years old. Consumers in the group of higher monthly income (NT\$40,001 50,000) emphasize the comfort attribute of shoes more than those consumers who have monthly income less than NT $\$ 20,000(\mathrm{~F}(4,417)=2.851$, $\mathrm{p}<.05)$. In addition, female consumers who are single tend to care shoes style $(F(2,419)=3.080, p<.05)$ and have higher purchase intention $(\mathrm{F}(2,419)=5.667, \mathrm{p}<.01)$ more than those married females consumers.

Table 4. Analysis of variance results for attributes and purchase intention of fashion shoes in demographics

\begin{tabular}{|c|c|c|c|c|c|c|c|c|}
\hline Attribute \& PI & Demographic & $\begin{array}{l}\text { Group } 1 \\
\text { Mean/SD }\end{array}$ & $\begin{array}{l}\text { Group } 2 \\
\text { Mean/SD }\end{array}$ & $\begin{array}{l}\text { Group } 3 \\
\text { Mean/SD }\end{array}$ & $\begin{array}{l}\text { Group } 4 \\
\text { Mean/SD }\end{array}$ & $\begin{array}{l}\text { Group } 5 \\
\text { Mean/SD }\end{array}$ & $F$ value & $p$ \\
\hline \multirow[t]{2}{*}{ Style } & Age & $18-25 \mathrm{yrs}$ & $26-35 \mathrm{yrs}$ & $36-45 \mathrm{yrs}$ & $>46 \mathrm{yrs}$ & & $F(3,418)$ & $.005^{* *}$ \\
\hline & Grp2 $>3$ & $6.32 / .75$ & $6.50 / .72$ & $6.11 / 1.20$ & $6.21 / 1.26$ & & $=4.320$ & \\
\hline \multirow[t]{2}{*}{ Style } & Marriage & Single & Married & Divorce & & & $F(2,419)$ & $.047^{*}$ \\
\hline & Grp1>2 & $6.42 / .74$ & $6.19 / 1.17$ & $6.43 / .535$ & & & $=3.080$ & \\
\hline \multirow[t]{2}{*}{ Colour } & Age & $18-25 y r s$ & $26-35 \mathrm{yrs}$ & $36-45 y r s$ & $>46 \mathrm{yrs}$ & & $\mathrm{F}(3,418)$ & $.001^{* *}$ \\
\hline & Grp2 $>3$ & $6.08 / .87$ & $6.39 / .68$ & $5.98 / 1.08$ & $6.18 / .91$ & & $=5.780$ & \\
\hline \multirow[t]{2}{*}{ Brand } & Age & $18-25 \mathrm{yrs}$ & $26-35 y r s$ & $36-45 \mathrm{yrs}$ & $>46 \mathrm{yrs}$ & & $\mathrm{F}(3,418)$ & $.010^{*}$ \\
\hline & Grp2>3 & $6.08 / 1.01$ & $6.30 / .84$ & $5.92 / 1.13$ & $6.29 / .71$ & & $=3.843$ & \\
\hline \multirow[t]{2}{*}{ Comfort } & Income & $<20 \mathrm{~K}$ & $20 \mathrm{~K}-30 \mathrm{~K}$ & $30 \mathrm{~K}-40 \mathrm{~K}$ & $40 \mathrm{~K}-50 \mathrm{~K}$ & $>50 \mathrm{~K}$ & $\mathrm{~F}(4,417)$ & $.024^{*}$ \\
\hline & Grp4>1 & $6.27 / .93$ & $6.48 / .68$ & $6.51 / .78$ & $6.73 / .49$ & $6.48 / 1.00$ & $=2.851$ & \\
\hline \multirow[t]{2}{*}{ PI } & Age & $18-25 y r s$ & $26-35 y r s$ & $36-45 y r s$ & $>46 \mathrm{yrs}$ & & $F(3,418)$ & $.000^{* * *}$ \\
\hline & Grp1,2>3,4 & $5.72 / .93$ & $5.83 / .99$ & $5.17 / 1.26$ & $4.85 / 1.51$ & & $=12.906$ & \\
\hline \multirow[t]{2}{*}{ PI } & Marriage & Single & Married & Divorce & & & $\mathrm{F}(2,419)$ & $.004^{* *}$ \\
\hline & Grp1>2 & $5.71 / 1.01$ & $5.33 / 1.28$ & $5.38 / 1.39$ & & & $=5.667$ & \\
\hline
\end{tabular}

Note. ${ }^{*} p<.05,{ }^{* *} p<.01,{ }^{* * *} p<.001$. 
Results in Table 5 suggested Hypothesis $3 b$ is partial supported, since the differences of consumer in shoes quantity, shoes purchased frequency, purchased price, and purchased locations had significant effectson style, brand name, comfort attributes and purchase intentions of fashion shoes. In general, the more shoes quantity consumers own $(\mathrm{F}(4,417)=23.161, \mathrm{p}<.001)$ and the higher shoes purchase frequency consumers have $(\mathrm{F}(4,417)=23.825, \mathrm{p}<.001)$, the higher purchase intentions of shoes would perceived. Precisely, females consumers who have more shoes quantity $(\mathrm{F}(4,417)=6.996, \mathrm{p}<.001)$ and have higher shoes purchased frequency $(\mathrm{F}(4,417)=2.675, \mathrm{p}<.05)$ tend to care shoes' style more than those who own less shoes quantity and have lower shoes shopping frequency. Additionally, females consumers who have more shoes quantity $(F(4,417)=5.715$, $\mathrm{p}<.001$ ) tend to care shoes' brand name more than those who own less shoes quantity. Females consumers who spend more on shoes purchased price tend to care more in shoes material $(\mathrm{F}(4,417)=6.738, \mathrm{p}<.001)$, collocability $(\mathrm{F}(4,417)=4.567, \mathrm{p}<.01)$ and comfort performance $(\mathrm{F}(4,417)=3.678, \mathrm{p}<.01)$. For the shoes purchase locations, consumer rank shoes sell in online stores have more style choice than retail stores and night market $(\mathrm{F}(4,417)=3.502, \mathrm{p}<.01)$; shoes sell in department stores have better materials than street vendors $(\mathrm{F}(4,417)=3.708, \mathrm{p}<.01)$, and better collocability presentation than those sell in night market $(\mathrm{F}(4,417)=4.467$, $\mathrm{p}<.01)$. Shoes sell in department stores and retail stores tend to have better wear comfort $(\mathrm{F}(4,417)=3.875$, $\mathrm{p}<.01)$ and service $(\mathrm{F}(4,417)=3.388, \mathrm{p}<.01)$ compare to those shoes sell in street vendors.

Table 5. Analysis of variance results for attributes and purchase intentionof shoes in shopping behavior

\begin{tabular}{|c|c|c|c|c|c|c|c|}
\hline $\begin{array}{l}\text { PI \& } \\
\text { Attribute }\end{array}$ & Shopping behavior & $\begin{array}{l}\text { Group } 1 \\
\text { Mean/SD }\end{array}$ & $\begin{array}{l}\text { Group } 2 \\
\text { Mean/SD }\end{array}$ & $\begin{array}{l}\text { Group } 3 \\
\text { Mean/SD }\end{array}$ & $\begin{array}{l}\text { Group } 4 \\
\text { Mean/SD }\end{array}$ & $\begin{array}{l}\text { Group } 5 \\
\text { Mean/SD }\end{array}$ & F value \\
\hline \multirow[t]{2}{*}{ PI } & Shoes Qty & 1 pairs & 2-4pairs & 5-7 pairs & 8-10 pairs & $>11$ pairs & $\mathrm{F}(4,417)$ \\
\hline & $\operatorname{Grp} 1<2<3,4,5$ & $3.98 / 1.18$ & $5.14 / 1.13$ & $5.68 / 1.03$ & $5.93 / .99$ & $6.03 / .94$ & $=23.161^{* * *}$ \\
\hline \multirow[t]{2}{*}{ PI } & Shoes purchase & a month & 3 months & 6 months & $6-12$ months & $>$ a year & $\mathrm{F}(4,417)$ \\
\hline & freq.Grp1,2>4>5 & $6.01 / .92$ & $5.91 / .94$ & $5.56 / .99$ & $4.95 / 1.29$ & $4.10 / 1.32$ & $=23.825^{* * *}$ \\
\hline \multirow[t]{2}{*}{ Style } & Shoes Qty & 1 pairs & 2-4pairs & 5-7 pairs & $8-10$ pairs & $>11$ pairs & $\mathrm{F}(4,417)$ \\
\hline & Grp $1,2<4,5$ & $5.75 / 1.44$ & $6.08 / 1.08$ & $6.37 / .80$ & $6.53 / .69$ & $6.60 / .79$ & $=6.996^{* * *}$ \\
\hline \multirow[t]{2}{*}{ Style } & Shoes purchase & a month & 3 months & 6 months & $6-12$ months & $>$ a year & $\mathrm{F}(4,417)$ \\
\hline & freq.Grp1,3>5 & $6.52 / .71$ & $6.36 / .94$ & $6.38 / .77$ & $6.24 / 1.00$ & $5.81 / 1.58$ & $=2.675^{*}$ \\
\hline \multirow[t]{2}{*}{ Brand } & Shoes Qty & 1 pairs & 2-4pairs & 5-7 pairs & 8-10 pairs & $>11$ pairs & $\mathrm{F}(4,417)$ \\
\hline & Grp $1<4,5$ & $5.78 / .94$ & $5.87 / 1.09$ & $6.19 / .89$ & $6.37 / .72$ & $6.37 / .98$ & $=5.715^{* * *}$ \\
\hline \multirow[t]{2}{*}{ Material } & Shoes price & $<1,500$ & $1,501-2,500$ & $2,501-3,500$ & $3,501-4,500$ & $>4,501$ & $\mathrm{~F}(4,417)$ \\
\hline & Grp $1,2<4$ & $5.87 / 1.07$ & $6.21 / .93$ & $6.47 / .73$ & $6.71 / .47$ & $6.42 / .669$ & $=6.738^{* * *}$ \\
\hline \multirow[t]{2}{*}{ Collocability } & Shoes price & $<1,500$ & $1,501-2,500$ & $2,501-3,500$ & $3,501-4,500$ & $>4,501$ & $\mathrm{~F}(4,417)$ \\
\hline & Grp $1<3,5$ & $4.18 / 1.29$ & $4.58 / 1.35$ & $4.88 / 1.15$ & $4.43 / 1.34$ & $5.25 / 1.87$ & $=4.567^{* *}$ \\
\hline \multirow[t]{2}{*}{ Comfort } & Shoes price & $<1,500$ & $1,501-2,500$ & $2,501-3,500$ & $3,501-4,500$ & $>4,501$ & $\mathrm{~F}(4,417)$ \\
\hline & Grp $1<3,4$ & $6.33 / .812$ & $6.49 / .80$ & $6.74 / .48$ & $6.79 / .43$ & $6.50 / .91$ & $=3.678^{* *}$ \\
\hline \multirow[t]{2}{*}{ Style } & Locations & Dept.store & Retail store & Street vendors & Onlinestore & Night market & $\mathrm{F}(4,417)$ \\
\hline & Grp $2,5<4$ & $6.40 / .84$ & $6.15 / 1.08$ & $6.42 / .90$ & $6.61 / .66$ & $5.88 / 1.36$ & $=3.502^{* *}$ \\
\hline \multirow[t]{2}{*}{ Material } & Locations & Dept. store & Retail store & Street vendors & Online store & Night market & $\mathrm{F}(4,417)$ \\
\hline & $\operatorname{Grp} 1<3$ & $6.31 / .93$ & $6.06 / .98$ & $5.50 / 1.24$ & $6.00 / .89$ & $5.75 / 1.28$ & $=3.708^{* *}$ \\
\hline \multirow[t]{2}{*}{ Collocability } & Locations & Dept. store & Retail store & Street vendors & Online store & Night market & $\mathrm{F}(4,417)$ \\
\hline & Grp $1<5$ & $4.71 / 1.34$ & $4.37 / 1.34$ & $4.00 / .85$ & $4.31 / 1.29$ & $3.13 / 1.25$ & $=4.467^{* *}$ \\
\hline \multirow[t]{2}{*}{ Comfort } & Locations & Dept. store & Retail store & Street vendors & Online store & Night market & $\mathrm{F}(4,417)$ \\
\hline & Grp $1,2>3$ & $6.56 / .73$ & $6.48 / .79$ & $5.75 / .97$ & $6.33 / .80$ & $6.38 / .52$ & $=3.875^{* *}$ \\
\hline \multirow[t]{2}{*}{ Service } & Locations & Dept. store & Retail store & Street vendors & Online store & Night market & $\mathrm{F}(4,417)$ \\
\hline & Grp $1,2>3$ & $5.55 / 1.27$ & $5.58 / 1.19$ & $4.50 / 1.17$ & $5.13 / 1.26$ & $5.38 / 1.19$ & $=3.388^{*}$ \\
\hline
\end{tabular}

Note. ${ }^{*} p<.05, * * p<.01,{ }^{* * *} p<.001$.

\section{Conclusion and Managerial Implication}

\subsection{Shoes Attributes}

According to the findings, comfort, style, colour, materials and collocability attributes of shoes are the most desire attributes for female consumers in Taiwan. Meanwhile, even though "comfort, durability, and service" are important attributes in shoes market from most consumers' and shoes manufactures perspective, these factors are the minimum essential attributes of shoes; the "style, colour, collocability, material, and brand name" are the real 
motivated attributes trigger female consumers' purchase intentions of shoes. This research identified the important trigger attributes of female shoes that they use most when purchasing shoes. It can provide retailers with a tool for aligning in female fashion shoes market. While shoes manufacturers focusing on comfort, durability, and materials attributes of shoes, they must also strive to establish designs in style and colour; increase collocability with fashion products; and build a positive brand image to achieve consumers' ultimate buying.

\subsection{Attitude, Subjective norms, and Perceived Behavior Control}

In addition, this research provides valuable insights for fashion shoes retailers by indicating consumers' purchase intentions would affect by different extent of attitude, subjective norms and perceived behavior control of consumers. The findings of this study suggests that creating a positive attitude toward purchasing fashion shoes may be a significant consideration for retailers to increase consumers' purchase intentions of fashion shoes. In order to cultivate favorable attitudes, footwear retailers should invest resources in shopping circumstances and atmosphere to create shopping value of excitement and pleasure which would eventually enhance consumers' attitude. In other hand, to increase the idea of social norms acceptance, footwear retailers could develop advertisements to cultivate the idea by using family and friends' alliance image. Latterly, footwear retailers who wish to improve consumer perceived behavior control should imply the affordable price image and affluent information of shoes in different media for consumers' resource.

\subsection{Demographic and Shopping Behavior Variables}

In demographic part, results identify two prominent segmentations: 18-35 years old single female consumers versus 36 to over 45 years old married female consumers. This single youth females group has higher purchase intentions of shoes and tends to care the style and colour attributes of shoes more than those elder married females. This youth group comprises college students and fresh employees in the job market. Unlike married females, most consumers in this group don't have pressures in financial and have personal time to shopping. They enjoy shopping and have supreme purchase power. Therefore, footwear retailers might want to target this niche market and study what is their favor design in style and colour. From the shopping behavior part, results indicated the purchase intentions of shoes is significant different from those who own more shoes quantity and have higher shoes purchase frequency (heavy user/buyer) than those who have own less shoes quantity and have lower shoes purchase frequency. The more pairs of shoes consumers own, the more they care about the brand name of shoes. Therefore, to increase sales and obtain higher market share, shoes retailers need to build and promote their brand image. Footwear retailers could use well-known starsas spokesman and integrate marketing communications skills to explore their brand image to consumers. Besides, results indicated the higher price consumers paid for shoes, the better material, collocability and comfort they care for the shoes. It is about the comprehensive value consumer perceived. If consumers spend more on shoes prices, of course they will like to receive a high quality of shoes. Footwear retailers that are target in luxury market need to pay more attention in material, collocability and comfort of shoes. Finally, since findings indicated that shoes distribution in different locations have significant differences of consumers appraise in style, material, collocability, comfort and service. Consumers tend to give higher appraise in department stores related to material, collocability, comfort and service, but not the style. Shoes retailers need to launch their merchandise carefully according to their target market.

\section{Limitations and Future Research}

Although the present findings provide a valuable insights for fashion shoes retailer, several suggestions for future research are made due to the study's limitations. First, the majority of study participants used in this research was female between the ages 18-45. Although female ages 18-45 is an appropriate age demographic for fashion shoes purchase behavior, future studies might examine whether differences exist between other age groups or gender in overall evaluations. In addition, this research is limited to female shoes category, findings may only be applicable to fashion merchandise categories; further study needs to obtain greater generalizability with variety of product categories. Additionally, since the mall-intercept method was used to collect survey data from Kaohsiung, a southern city in Taiwan, the findings of this study may not be applicable to all consumers in Taiwan or elsewhere. Thus future research could incorporate online survey method which would allow samplings from various geographic locations. Finally, this study can be extended to be an international comparative study. Study found in this research shows attitude, subjective norms and perceived behavior control made significant differences between high and low purchase intentions of shoes in Taiwan, it is important to explore the effect of attitude, subjective norms, and perceived behavioral controlon purchase intention varies in different cultures. 


\section{References}

Aaker, D. A. (1991). Managing brand equity: Capitalizing on the value of a brand name. New York, NY: The Free Press.

Aaker, D. A., Batra, R., \& Myers, J. G. (1992). Advertising management (4th ed.). London: Prentice Hall.

Ajzen, I. (1991). The theory of planned behavior. Organizational Behavior and Human Decision Processes, 50(2), 179-211. http://dx.doi.org/10.1016/0749-5978(91)90020-T

Akpoyomare, O. B., Adeosun, L. P., \& Ganiyu, R. A. (2012). The influence of product attributes on consumer purchase decision in the Nigerian food and beverages industry: Astudy of Lagos Metropolis. American Journal of Business and Management, 1(4), 196-201.

Armitage, C. J., \& Conner, M. (2001). Efficacy of the theory of planned behaviour: A meta-analytic review. British Journal of Social Psychology, 40, 471-499. http://dx.doi.org/10.1348/014466601164939

Bashirian, S., Hidarnia, A., Allahverdipour, H., \& Hajizadeh, E. (2012). Application of the theory of planned behavior to predict drug abuse related behaviors among adolescent. Journal of Research in Health Sciences, $12,54-60$.

Belch, G. E., \& Belch, M. A. (2001). Advertising and Promotion: an Integrated Marketing Communications Perspective (5th ed.). Boston, Mass: Irwin/McGraw-Hill.

Bhattacherjee, A. (2000). Acceptance of e-commerce services: The case of electronic brokerages. IEEE Transactions on Systems, Man, and Cybernetics- Part A: Systems and Humans, 30(4), 411-420. http://dx.doi.org/10.1109/3468.852435

Canniere, M. H., Pelsmacker, P., \& Geuens, M. (2009). Relationship quality and the theory of planned behavior models of behavioral intentions and purchase behavior. Journal of Business Research, 62, 82-92. http://dx.doi.org/10.1016/j.jbusres.2008.01.001

Chang, C. Y., Wang, J. M., \& Cheng, H. W. (2010). A study of customer loyalty on sport shoes. Journal of Leisure, Tourism, Sport, \& Health, 1(1), 14-32.

Culiberg, B. (2014). Towards an understanding of consumer recycling from an ethical perspective. International Journal of Consumer Studies, 38, 90-97. http://dx.doi.org/10.1111/ijcs.12068

Davis, R. A., Lang, B., \& San Diego, J. (2014). How gender affects the relationship between hedonic shopping motivation and purchase intentions? Journal of Consumer Behaviour, 13(1), 18-30. http://dx.doi.org/10.1002/cb.1450

Eagly, A. H., \& Chaiken, S. (1993). The psychology of attitudes. San Diego, CA: Harcourt Brace Jovanovich.

Elliott, M. A., Armitage, C. J., \& Baughan, C. J. (2007). Using the theory of planned behaviour to predict observed driving behaviour. British Journal of Social Psychology, 46, 69-90. http://dx.doi.org/10.1348/014466605X90801

Executive Yuan, R. O. C. (2013). Directorate-General of Budget, Accounting and Statistics, general statistic news \#043. Retrieved from http://www.civil.taichung.gov.tw/public/Attachment/1/33118525190.pdf

Forney, J., Park, E. J., \& Brandon, L. (2005). Effects of evaluative criteria on fashion brand extension. Journal of Fashion Marketing and Management, 9, 156-165. http://dx.doi.org/10.1108/13612020510599312

Gobi International. (2010). World business information-Footwear. Retrieved from http://www.gobi.co.uk/miscellaneous.htm

Head, M., \& Ziolkowski, N. (2012). Understanding student attitudes of mobile phone features: Rethinking adoption through conjoint, cluster and SEM analyses. Computers in Human Behavior, 28, 2331-2339. http://dx.doi.org/10.1016/j.chb.2012.07.003

Hedrick-Wong, Y. W. (2006). Holding up half of the sky: The new women consumers of Asia. Singapore: John Wiley \& Sons.

Industrial Development Bureau, R. O. C. (2011). 2011 Global footwear markets development and shoes material trends analysis, Ministry of Economic Affairs. Retrieved from http://www.tipo.org.tw/docs/fup/2_2011\%E5\%85\%A8\%E7\%90\%83\%E9\%9E\%8B\%E6\%A5\%AD\%E7\%9 9\%BC\%Е5\%B1\%95\%Е5\%80\%8B\%Е8\%AB\%96\%Е8\%88\%87\%Е9\%9Е\%8B\%Е6\%9D\%90\%Е8\%B6\%A $8 \% \mathrm{E} 5 \% 8 \mathrm{~B} \% \mathrm{~A} 2 \% \mathrm{E} 5 \% 88 \% 86 \% \mathrm{E} 6 \% 9 \mathrm{E} \% 90$.pdf 
Kim, H., \& Karpova, E. (2010). Consumer attitudes toward fashion counterfeits: application of the theory of planned behavior. Clothing and Textiles Research Journal, 28, 79-94. http://dx.doi.org/10.1177/0887302X09332513

Kotler, P. (2002). Marketing management (11th ed.). Upper Saddle River, NJ: Pearson Education.

Laiwechpittaya, T., \& Udomkit, N. (2012). A matter of shoes: The analysis of desired attributes of shoes and its retail shops from Bangkok consumers' perspectives. International Journal of Marketing Studies, 5, 33-40.

Lancaster, K. (1966). A new approach to consumer theory. Journal of Political Economy, 74, 132-157. http://dx.doi.org/10.1086/259131

Ling, G. (2009). Understanding consumer purchasing behavior regarding luxury fashion-related goods (Unpublished doctoral dissertation). The Hong Kong Polytechnic University. Retrieved from http://repository.lib.polyu.edu.hk/jspui/bitstream/10397/3954/2/b23216323_ir.pdf

Saha, S., Dey, M., \& Bhattacharyya, S. (2010). Factors affecting consumer buying behavior of Shoes in Kolkata: A case study. The IUP Journal of Management Research, 9, 39-60.

Shaw, D., \& Shiu, E. (2002). An assessment of ethical obligation and self-identity in ethical consumer decision-making: A structural equation modelling approach. International Journal of Consumer Studies, 26, 286-293. http://dx.doi.org/10.1046/j.1470-6431.2002.00255.x

Stanton, W. J., Michael, J. E., \& Bruce, J. W. (1991). Fundamentals of marketing (9th ed.). New York, NY: McGraw-Hill.

Topa, G., \& Moriano, J. A. (2010). Theory of planned behavior and smoking: meta-analysis and SEM model. Substance Abuse and Rehabilitation, 1, 23-33. http://dx.doi.org/10.2147/SAR.S15168

\section{Copyrights}

Copyright for this article is retained by the author(s), with first publication rights granted to the journal.

This is an open-access article distributed under the terms and conditions of the Creative Commons Attribution license (http://creativecommons.org/licenses/by/3.0/). 